\title{
ON OPTIMAL DISTRIBUTION OF OUTPUT FROM A JOINTLY OWNED RESOURCE
}

\author{
David GALE and Joel SOBEL \\ University of California, La Jolla, CA 92093, USA
}

Received June 1980, accepted December 1980

\begin{abstract}
A resource is owned jointly by $m$ agents, the $i$ th agent's share of the resource being $\theta_{i}$. The output of the resource, and the utilities of each agent are functions of the state of nature. An admissible distribution scheme is one which (1) is (Pareto) optimal and which (2) gives each agent an expected consumption proportional to his share of the resource. We show that with the usual concavity assumptions on utilities there always exists one and only one admissible distribution scheme. The proof is achieved by constructing a suitable social welfare function which is maximized at the desired distribution scheme.
\end{abstract}

\section{Introduction}

This study deals with situations of the following sort. A resource is owned jointly by a number of agents, each agent's share being some exogenously specified number. The prototypical example would be a firm owned by stockholders. The resource produces some homogeneous output (profit) which is assumed to be a random variable with known distribution. The problem is then to determine some rule for distributing the output among the agents which is consistent with their ownership shares. Such a rule will be called a distribution scheme.

The situation described above may be though of as a special case of the much studied bargaining problem in which a number of agents are faced with a set of possible outcomes. An outcome which is unanimously agreed to is implemented. If the agents fail to agree some prescribed disagreement. outcome results.

The modern theory of this subject probably begins with the work of Nash (1950). The Nash theory is not concerned with the actual negotiations among agents (as the word bargaining might suggest) but is interested only in determining some rule for redistribution which satisfies certain conditions, or axioms, which would presumably be acceptable to the bargainers or required by the situation. The main result of the theory is a theorem asserting the existence of a unique redistribution rule satisfying the given 0304-4068/82/0000-0000/\$02.75 (C) 1982 North-Holland 
axioms. The Nash formulation has often been criticized, however, because it depends only on the set of possible utility vectors achievable by the agents and not on the underlying situation which gave rise to the original problem.

In analyzing our distribution problem we have followed the Nash approach. The notable feature in this special case is that the requirements (axioms) are extremely simple. We require only (1) the scheme should be weakly Pareto optimal, meaning that no other scheme should give every agent a greater expected utility, and (2) each agent's expected share of output should be proportional to his share of ownership. Notice that although (1) depends on considerations of utility it is perhaps the weakest such requirement one could make, and (2) is completely independent of any considerations of utility depending only on the objective data of the problem, namely the random output and the relative shares of ownership. Our result asserts that, assuming agents have smooth concave utility functions, there is exactly one distribution scheme consistent with (1) and (2). Moreover, like the Nash bargaining solution the distribution rule is characterized as the solution to a constrained maximum problem.

A few further remarks may help to illuminate the nature of the problem:

Remark 1. This investigation was originally motivated by the observation that the 'obvious' solution to the problem, namely, give each agent a share of output proportional to his share of ownership will almost always violate Pareto optimality. This is not hard to see. Consider for example the simple case of the two agents owning equal shares of a firm, but having different utilities for income, suppose there are only two states of nature, so the firm either makes or loses money. Then it will almost always be the case that a fifty-fifty split of profits or loss would be dominated by some scheme in which one agent agrees to accept less than half the profits in a good year in return for having to bear less than half the losses in a bad one.

Remark 2. It does not seem immediately clear that there even exist rules satisfying both (1) and (2) though this can be established by means of fixed point arguments. The uniqueness can also be given a separate proof using semi-combinatorial methods. This approach is in fact used by the second author in a related paper [Sobel (1981)].

Remark 3. The model we treat allows an agent's utility function as well as output to depend on the state of nature. This generalization has a natural interpretation. Thus, if the state of nature was such that an agent (stockholder) suffered some serious financial reverse (say, his house burned down) then his utility for income would presumably be higher than normal. This interpretation has in fact been exploited in Bühlmann and Jewell (forthcoming $\mathrm{a}, \mathrm{b}$ ) which are concerned with insurance pooling or 'risk exchange'. 
Remark 4. Our solution to this distribution problem possesses many of the properties required of bargaining solutions for other models. Specifically the axioms of Pareto optimality and invariance with respect to positive affine transformations are satisfied by our solution. Further the solution is symmetric when the shares of the agents are equal. Symmetry, invariance with respect to positive affine transformations and Pareto optimality are the axioms common to the Nash (1950) and the Raiffa (1953) and KalaiSmorodinsky (1975) theories of bargaining.

\section{The model and the social welfare function}

We formalize the problem described in the introduction. The given parameters of the problem are:

(i) there are $m$ agents $A_{i}$ for $i=1,2, \ldots, m$;

(ii) a non-negative, bounded, measurable function $h$ (the amount of output) defined on a probability space $S$ (the states of nature);

(iii) real-valued utility functions $u_{i}$ defined on $S \times R$ for $i=1, \ldots, m$.

We write $u_{i}(s, x)$ and assume the $u_{i}$ are increasing, strictly concave, and differentiable in $x$. Letting $u_{i}^{\prime}$ denote differentiation with respect to $x$, we assume $u_{i}$ and $u_{i}^{\prime}$ are bounded and measurable in $s$. [The quantity $u_{i}(s, x)$ measures the satisfaction to $A_{i}$ of receiving $x$ in state $s$.]

(iv) positive numbers $\theta_{1}, \ldots, \theta_{m}$ summing to 1 (the shares of the $m$ agents).

A distribution scheme is a vector-valued non-negative measurable function $d=\left(d_{1}, \ldots, d_{m}\right)$ on $S$, where $d_{i}(s)$ represents the amount distributed to $A_{i}$ in state $s$. We denote by $D$ the set of all distribution schemes. The scheme $d$ in $D$ is called feasible if

$$
\sum d_{i}(s)=h(s) \quad \text { for all } s .
$$

If in addition we have

$$
\int_{S} d_{i}(s)=\theta_{i} \int_{S} h(s) \quad \text { for all } i
$$

we say that $d$ is proportional. ${ }^{1}$

Note that the set of all proportional schemes forms a convex subset of $L_{\infty}(S)^{m}$ (bounded measurable functions from $S$ to $R^{m}$ ).

\footnotetext{
${ }^{1}$ Throughout the paper integration over $S$ of function $f(s, \cdot)$ will be denoted by $\int_{S} f(s, \cdot)$.
} 
For each $d$ in $D$ we define the $m$-vector $u(d)$ by

$$
u(d)=\left(\int_{s} u_{1}\left(s, d_{1}(s)\right), \ldots, \int_{S} u_{m}\left(s, d_{m}(s)\right)\right)
$$

Thus, $u(d)$ is the set of expected utilities of the $A_{i}$ 's under the scheme $d$. A feasible scheme $d$ is called optimal if there is no other feasible scheme $d$ such that $u(d)>u(d)$ (we use the usual vector inequality notation).

We now define the social welfare function $\Phi$. First, define $\phi_{i}$ on $S \times R_{+}$by

$$
\phi_{i}(s, x)=\int_{i}^{x} \log u_{i}^{\prime}(s, t) \mathrm{d} t,
$$

and note that $\phi_{i}$ is differentiable and strictly concave in $x$ since

$$
\phi_{i}^{\prime \prime}(s, x)-u_{i}^{\prime \prime}(s, x) / u_{i}^{\prime}(s, x),
$$

which is negative from the assumptions on the $u_{i}$.

Now for any $d$ in $D$ define

$$
\Phi(d)=\sum_{i=1}^{m} \int_{S} \phi_{i}\left(s, d_{i}(s)\right) .
$$

Observe that $\Phi$ is a strictly concave function on $D$ so that it achieves at most one maximum on any convex set.

To prove our main result we will show:

Theorem 1. A distribution scheme is proportional and optimal if and only if it maximizes $\Phi$ among all proportional distribution schemes.

The analysis to follow hinges critically on the particular functions $\phi_{i}$ we have chosen. It would be illuminating to have some economic interpretation of these functions. In that direction $\mathrm{H}$. Bühlmann has observed that the quantity on the right of (2.3) is the negative of the Arrow (1971) and Pratt (1964) measure of 'risk aversion'. In fact, any second integral of the risk aversion would do as well as the $\phi_{i}$ for suppose $\tilde{\phi}_{i}(s, x)=\phi_{i}(s, x)+a_{i} x+b_{i}$, and let $\widetilde{\Phi}$ be the corresponding integral as in (2.4). Then

$$
\int_{S} \tilde{\phi}_{i}\left(s, d_{i}(s)\right)=\int_{S} \phi_{i}\left(s, d_{i}(s)\right)+a_{i} \int_{S} d_{i}(s)+b_{i},
$$


but by (2.2) the second term on the right is $a_{i} \theta_{i} \int_{s} h(s)$, so

$$
\tilde{\Phi}(d)=\Phi(d)+\left(\sum a_{i} \theta_{i}\right) \int_{S} h(s)+\sum b_{i},
$$

and so $d$ maximizes $\Phi$ if and only if it maximizes $\tilde{\Phi}$.

In the next section we will prove the existence of a welfare maximizing proportional distribution scheme. Section 4 will present the needed results from optimization theory (Kuhn-Tucker theorems) and these will be applied in section 5 to prove Theorem 1.

\section{Existence of a welfare-maximizing proportional distribution scheme}

We endow the set $D$ with the weak-star topology. This means that $d$ is a limit point of the sequence $d^{n}$ if for any function $f$ in $L_{1}^{m}(S), \lim _{n \rightarrow \omega} \int_{S}\left(d_{i}(s)\right.$ $\left.-d_{i}^{n}(s)\right) f(s)=0$.

Let $D_{p}$ be the set of proportional distribution schemes. From the feasibility condition (2.1) $D_{p}$ is bounded. It is also an easy verification to show that $D_{p}$ is weak-star closed and hence, by a well-known result, compact [see, e.g., Lang (1969)]. Now let $\sigma=\sup \left\{\Phi(d) \mid d \in D_{\mathrm{p}}\right\}$. By compactness of $D_{\mathrm{p}}$ there is a function $\bar{d}$ in $D_{\mathrm{p}}$ and a sequence $d^{n} \rightarrow \bar{d}$ such that $\Phi\left(d^{n}\right) \rightarrow \sigma$. We claim that $\Phi(\bar{d})=\sigma$ for

$$
\Phi(d)-\Phi\left(d^{n}\right)=\sum_{i} \int_{S}\left(\Phi_{i}\left(s, d_{i}(s)\right)-\phi_{i}\left(s, d_{i}^{n}(s)\right)\right)
$$

but by concavity of the $\phi_{i}$ we have

$$
\phi_{i}\left(s, d_{i}(s)\right)-\phi_{i}\left(s, d_{i}^{n}(s)\right) \geqq \phi_{i}^{\prime}\left(s, d_{i}(s)\right)\left(d_{i}(s)-d_{i}^{n}(s)\right),
$$

so, since $\phi_{i}^{\prime}\left(s, d_{i}(s)\right)$ is in $L_{1}(S)$ (from our assumption on the $u_{i}$ ), we have

$$
\lim _{n \rightarrow \infty} \int_{S} \phi_{i}^{\prime}\left(s, \bar{d}_{i}(s)\right)\left(d_{i}(s)-d_{i}^{n}(s)\right)=0,
$$

by weak-star convergence. Combining (3.1), (3.2) and (3.3) gives

$$
\lim _{n \rightarrow \infty}\left(\Phi(d)-\Phi^{n}(d)\right) \geqq 0 \quad \text { so } \quad \Phi(\bar{d})=\sigma .
$$

\section{Theorems on constrained maximization}

We shall need two lemmas on constrained maximization. The first is a simple infinite dimensional version of the Kuhn-Tucker Theorem. 
Let $\Phi$ be a concave function defined on a convex set $K$ in a real linear space $X$. Let $L$ be a linear function from $X$ to $R^{m}$, and assume for each $y$ in $L(K)$ that the function $\Phi$ attains a maximum on $L^{-1}(y)$. Let $b$ be in the relative interior of $L(K)$.

Lemma 1 (Kuhn-Tucker). The point $\bar{x}$ maximizes $\Phi(x)$ subject to $x \in K$, and $L(x)=b$ if and only if there exists an m-vector $v$ such that $\bar{x}$ maximizes $\Phi(x)-v \cdot L(x)$ for $x$ in $K$.

The sufficiency of the condition is immediate and the necessity follows just as in the finite-dimensional case as proved e.g. in Gale (1968). Namely, for $y$ in $L(K)$ define $\mu(y)=\max _{L(x)=y} \Phi(x)$ and verify that $\mu$ is concave in $y$. The fact that $b$ is in the relative interior of $L(K)$ means that $\mu$ has a support (or super gradient) at $b$, thus, there is an $m$-vector $v$ such that $\mu(y)-\mu(b) \leqq v \cdot(y-b)$ for all $y$ in $L(K)$. One easily verifies that $v$ satisfies the conclusion of the lemma.

Next let $f_{i}(s, x), i=1, \ldots, m$, be functions on $S \times R_{+}$where $S$ is a probability space, and assume each $f_{i}$ is differentiable and strictly concave in $x$ and $f_{i}$ and $f_{i}^{\prime}$ are bounded and measurable on $S \times R$. Let $h$ be a bounded measurable function on $S$. In the class of non-negative bounded measurable functions $\mu=\left(\mu_{1}, \ldots, \mu_{m}\right)$ from $S$ to $R_{+}^{m}$ consider the problem:

(I) Find a function $\mu$ which maximizes

$$
\Phi(\mu)=\left(\int_{s} \sum_{i} f_{i}\left(s, \mu_{i}(s)\right)\right)
$$

subject to

$$
\sum \mu_{i}=h
$$

Lemma 2. The function $\bar{\mu}$ solves (П) if and only if there exists a function $g$ on $S$ such that, for almost all s,

$$
\begin{aligned}
f_{i}^{\prime}\left(s, \bar{\mu}_{i}(s)\right) & \leqq g(s), \\
& =g(s) \text { if } \quad \bar{\mu}_{i}(s)>0 .
\end{aligned}
$$

Let $\bar{\mu}$ solve (ח) and let $g(s)=\max _{i} f_{i}^{\prime}\left(s, \mu_{i}(s)\right)$. Suppose (4.3) does not hold. 
Then there is a set $S_{1}$ of positive measure, an $\varepsilon>0$, and a pair of indices, say 1 and 2 , such that for all $s$ in $S_{1}$,

(i)

$$
\mu_{1}(s) \geqq \varepsilon,
$$

(ii)

$$
f_{1}^{\prime}\left(s, \mu_{1}(s)\right)<f_{2}^{\prime}\left(s, \mu_{2}(s)\right) .
$$

We get a contradiction by showing that $\bar{\mu}$ does not maximize $\Phi$. For $\delta$ in $(0, \varepsilon)$ define $\mu_{\delta}$ by

$$
\begin{aligned}
& \mu_{\delta}(s)=\left(\bar{\mu}_{1}(s)-\delta, \bar{\mu}_{2}(s)+\delta, \bar{\mu}_{3}(s), \ldots, \bar{\mu}_{m}(s)\right) \text { for } s \in S_{1}, \\
& =\bar{\mu}(s) \quad \text { for } s \notin S_{1} .
\end{aligned}
$$

Clearly $\mu_{\delta}$ is bounded and measurable.

Let

$$
\begin{aligned}
q(\delta)= & \Phi\left(\mu_{\delta}\right)-\Phi(\bar{\mu}) \\
= & \int_{s}\left(\sum_{i=1}^{m} f_{i}\left(s, \mu_{\delta_{i}}(s)\right)-\sum_{i=1}^{m} f_{i}\left(s, \bar{\mu}_{i}(s)\right)\right) \\
= & \int_{s_{1}}\left(f_{1}\left(s, \bar{\mu}_{1}(s)-\delta\right)-f_{1}\left(s, \bar{\mu}_{1}(s)\right)-f_{2}\left(s, \bar{\mu}_{2}(s)\right)\right. \\
& \left.+f_{2}\left(s, \bar{\mu}_{2}(s)+\delta\right)\right) .
\end{aligned}
$$

Because of the hypotheses on the $f_{i}$ we may differentiate under the integral sign giving

$$
q^{\prime}(0)=\int_{s_{1}}\left(-f_{1}^{\prime}(s, \bar{\mu}(s))+f_{2}^{\prime}(s, \bar{\mu}(s))>0,\right.
$$

so for $\delta$ small $q(\delta)$ is positive, and so $\Phi\left(\mu_{\delta}\right)>\Phi(\bar{\mu})$ which is the desired contradiction.

Conversely, suppose (4.3) holds for some s. It then follows that $\mu(\bar{s})$ $=\left(\bar{x}_{1}, \ldots, \bar{x}_{m}\right)$ maximizes $\sum f_{i}\left(s, x_{i}\right)$ subject to $\sum x_{i}=h$. To see this, first note that (4.3) implies

$$
f_{i}^{\prime}\left(s, \bar{x}_{i}\right)\left(x_{i}-\bar{x}_{i}\right) \leqq g(s)\left(x_{i}-\bar{x}_{i}\right),
$$

for all $x_{i} \geqq 0$ (note $x_{i}<\bar{x}_{i}$ means $\bar{x}_{i}>0$ so $f_{i}\left(s, \bar{x}_{i}\right)=g(s)$ ). 
Next by concavity of $f_{i}$,

$$
f_{i}\left(s, x_{i}\right)-f_{i}\left(s, \bar{x}_{i}\right) \leqq f_{i}^{\prime}\left(s, \bar{x}_{i}\right)\left(x_{i}-\bar{x}_{i}\right),
$$

so we get

$$
\sum f_{i}\left(s, x_{i}\right)--\sum f_{i}\left(s, \bar{x}_{i}\right) \leqq \sum g(s)\left(x_{i}-\bar{x}_{i}\right)
$$

but if $\sum x_{i}=h$, the right side above is zero. This shows that (4.3) implies that $\bar{\mu}(s)$ maximizes the integrand of (4.1) for almost all $s$, and hence the integral (4.1) is also maximized.

\section{Proof of Theorem 1}

Let $d$ maximize $\Phi$. Let $\hat{D}$ be the set of all feasible distribution schemes and define $L$ on $\hat{D}$ by

$$
L(d)=\left(\int_{S} d_{1}(s), \ldots, \int_{S} d_{m}(s)\right) .
$$

Note that $L(\hat{D})$ consists of all non-negative vectors $y=\left(y_{1}, \ldots, y_{m}\right)$ such that $\sum_{i=1}^{m} y_{i}=h$. Since all $\theta_{i}$ are positive, the point $\left(\theta_{1} h, \ldots, \theta_{m} h\right)$ is in the relative interior of $L(\hat{D})$, so the Kuhn-Tucker Theorem implies there exists an $m$ vector $v$ such that $d$ maximizes $\Phi(d)-v \cdot L(d)$ for $d$ in $\hat{D}$. Now applying Lemma 2 to the case where the functions $f_{i}(s, x)=\phi_{i}(s, x)-v_{i} x$ $=\int_{1}^{x} \log u_{i}^{\prime}(s, c) \mathrm{d} c-v_{i} x$, we get the existence of a function $g$ such that

$$
\log u_{i}^{\prime}\left(s, d_{i}(s)\right)-v_{i} \leqq g(s),
$$

with equality if $\bar{d}_{1}(s)>0$ for almost all $s$. Letting $\alpha_{i}=\mathrm{e}^{-v_{i}}$ and $\beta(s)=\mathrm{e}^{g(s)}$, we get

$$
\alpha_{i} u_{i}^{\prime}\left(s, \bar{d}_{i}(s)\right) \leqq \beta(s)
$$

with equality if $d_{i}(s)>0$.

Applying Lemma 2 in the other direction shows that $d$ maximizes $\sum \alpha_{i} u_{i}\left(s, d_{i}(s)\right)$ subject to $\sum d_{i}(s)=h(s)$. Therefore if $d$ is in $\hat{D}$ we have

$$
\sum \alpha_{i} u_{i}\left(s, \bar{d}_{i}(s)\right) \geqq \sum \alpha_{i} u_{i}\left(s, d_{i}(s)\right),
$$

for almost all $s$, so

$$
\sum \alpha_{i} \int_{s} u_{i}\left(s, \bar{d}_{i}(s)\right) \geqq \sum \alpha_{i} \int_{s} u_{i}\left(s, d_{i}(s)\right),
$$


for all $d$ in $\hat{D}$, so since $\alpha_{i}>0$ for all $i$ there cannot be $d$ in $\hat{D}$ with $\int_{S}\left(u_{i}(s, d(s))\right.$ $\left.-u_{i}\left(s, d_{i}(s)\right)\right) \geqq 0$ with inequality holding for some $i$, and therefore $d$ is optimal.

Conversely, suppose $d$ is proportional and optimal. Let $U$ be the set of all points $v$ in $R^{m}$ such that there exists a $d$ in $D$ with $u(d) \geqq v$. From concavity of the $u_{i}$ it follows that $U$ is convex and since $d$ is optimal $u(d)$ is an efficient point of $U$. By the standard efficiency theorem, therefore, there exist nonnegative numbers $\alpha_{i}$ such that

$$
\bar{d} \text { maximizes } \sum \alpha_{i} \int_{S} u\left(s, d_{i}(s)\right) \text {, }
$$

for $d$ in $D_{p}$.

In fact $\alpha_{i}>0$ for all $i$, for suppose $\alpha_{1}=0, \alpha_{2}>0$. Then since $\theta_{1}>0$ we must have $d_{1}(s)>0$ on some set $S_{1}$ of positive measure, but then (5.4) could be increased by decreasing $d_{1}$ and increasing $d_{2}$ on the set $S_{1}$ since the function $u_{2}$ is increasing in $x$.

Applying Lemma 2 to (5.4) gives the existence of a function $\beta$ such that, for almost all $s$,

$$
\alpha_{i} u_{i}\left(s, \bar{d}_{i}(s)\right) \leq \beta(s),
$$

with equality for $d_{i}(s)>0$. Since the $\alpha_{i}$ are positive so is $\beta(s)$. Setting $v_{i}=$ $-\log \alpha_{i}$ and $g(s)=\log \beta(s)$ we get (5.1), and by Lemma 2 once more and the Kuhn-Tucker Theorem in the easy direction we see that $d$ maximizes $\Phi$.

\section{References}

Arrow, K., 1971, The theory of risk aversion, in: Essays in the theory of risk bearing, Ch. 2 (Markham, Chicago, IL).

Bühlmann, H. and W.S. Jewell, forthcoming a, Unicity of fair optimal risk exchange, Scandinavian Actuarial Journal.

Bühlmann, H. and W.S. Jewell, forthcoming b, Optimal risk exchange, ASTIN Bulletin.

Gale, D., 1968, A mathematical theory of optimal economic development, Bulletin of the American Mathematical Society 74, no. 2, 176-180.

Kalai, Ehud, 1977, Proportional solutions to bargaining structures: Interpersonal utility comparisons, Econometrica 45, 1623-1630.

Kalai, Ehud and Meir Smorodinsky, 1975, Other solutions to Nash's bargaining problem, Econometrica 43, 513-518.

Lang, Serge, 1969, Real analysis (Addison-Wesley, Reading, MA).

Nash, John F., 1950, The bargaining problem, Econometrica 28, $155-162$.

Pratt, J.W., 1964, Risk aversion in the small and in the large, Econometrica 32, 122-137.

Raiffa, Howard, 1953, Arbitration schemes for generalized two-person games, in: H.W. Kuhn and A.W. Tucker, Contributions to the theory of games II, Analysis of Mathematical Studics 28 (Princeton, NJ) 361-387.

Sobel, Joel, 1981, Proportional distribution schemes, Journal of Mathematical Economics $8,147-157$. 